\title{
Oscillatory Deformation of Chemical Waves Induced by Surface Flow
}

\author{
Hidetoshi Miike, (a) Stefan C. Müller, and Benno Hess \\ Max-Planck-Institut für Ernährungsphysiologie, Rheinlanddamm 201, D-4600 Dortmund 1, Federal Republic of Germany \\ (Received 13 April 1988)
}

\begin{abstract}
The dynamic behavior of chemical patterns induced by the coupling of chemical waves and hydrodynamic flows in the Belousov-Zhabotinskii reaction was investigated by $2 \mathrm{D}$ spectrophotometry and $2 \mathrm{D}$ velocimetry. Periodically varying deformations of chemical-wave profiles were observed in a reactive layer with a liquid/gas interface. They are due to an oscillatory hydrodynamic flow detected close to the layer surface.
\end{abstract}

PACS numbers: 47.70.Fw, 47.20.Dr, 82.20.Mj

Spontaneous pattern formation in far from equilibrium systems is of great current interest in many disciplines of science. A well-known example is the occurrence of circular and spiral-shaped chemical waves in the Belousov-Zhabotinskii (BZ) reaction. They are treated theoretically by modeling the nonlinear coupling of complex reaction kinetics and diffusion. ${ }^{1}$ The dish containing a thin solution layer is frequently covered in order to minimize evaporation, but if it is left uncovered, a variety of structural phenomena are observed, e.g., stationary "mosaic" patterns, transient structures, or distortion and decomposition of waves..$^{2-5}$ For an explanation, the influence of convective flows has been discussed, which arise from temperature gradients as a result of evaporative cooling and from inhomogeneities in surface tension as a result of differences in temperature and/or chemical composition (Rayleigh-Bénard and Marangoni-type instabilities). ${ }^{6,7}$ Frequently, a quantitative comparison is made with analogous patterns in hydrodynamically unstable simple liquids. ${ }^{6,8}$

Recently, we detected that hydrodynamic flow is associated with chemical-wave propagation both in covered and uncovered sample dishes. ${ }^{9}$ Remarkably, the flow becomes oscillatory, especially at a surface left open to evaporation and is possibly entrained by the periodic passage of waves. In this Letter we apply space-resolved spectrophotometry ${ }^{10}$ and velocimetry ${ }^{9}$ to areas of typically $3 \times 3 \mathrm{~mm}^{2}$ of wave patterns in an excitable BZ solution layer. We report on periodically changing deformations of wave profiles and fronts. It is clarified that the deformations are not induced by convection in the bulk of the liquid but by oscillatory hydrodynamic flow near the surface.

The preparation of an excitable $\mathrm{BZ}$ solution has been previously described. ${ }^{11}$ For velocity measurements, after filtering the solution through $0.22-\mu \mathrm{m}$ filter, a small amount of polystyrene latex particles (diameter 0.48 $\mu \mathrm{m}$ ) serving as scattering centers were added. A volume of the mixture was placed in a dust- and scratch-free Petri dish at $25 \pm 1{ }^{\circ} \mathrm{C}$. To obtain a constant period of wave propagation, a pair of spiral waves was initiated ${ }^{11}$ (Fig. 1). Time of the experiments started $(t=0)$ briefly afterwards. The spiral centers were located about $2 \mathrm{~cm}$ away from the dish center, where measurements were carried out. Note that no $\mathrm{CO}_{2}$ bubbles were nucleated and no waves were triggered at the dish boundaries. Patterns were investigated with and without covering the dish (air gap between layer surface and glass cover $\approx 12$ $\mathrm{mm}$ ).

The correlation between wave patterns and hydrodynamics was investigated by spectrophotometry and velocimetry based on 2D microscope-video-imaging techniques. ${ }^{9-11}$ A He-Ne laser focused to a small area (diameter $0.2 \mathrm{~mm}$ ) illuminated the polystyrene particles, thus allowing the detection of local hydrodynamic flow. The microscope focal plane was usually adjusted close to the layer surface. Flow velocity was measured every $5 \mathrm{~s}$ by pursuing one or two particles during $1 \mathrm{~s}$ on a video movie displayed on a television monitor. By our also shining a homogeneous light field $(490 \mathrm{~nm})$ through the layer, particle motion and propagation of chemical activity could be traced simultaneously. ${ }^{9}$ In addition, video movies of monochomatic transmitted light without illuminated polystyrene particles were recorded to observe the dynamic characteristic of the chemical waves. Local time traces of light absorption were obtained by placing a photodiode in front of a selected spot on the monitor (corresponding to a diameter $<20 \mu \mathrm{m}$ ), and the local luminescence of the displayed image sequence was measured and analyzed by a computer.

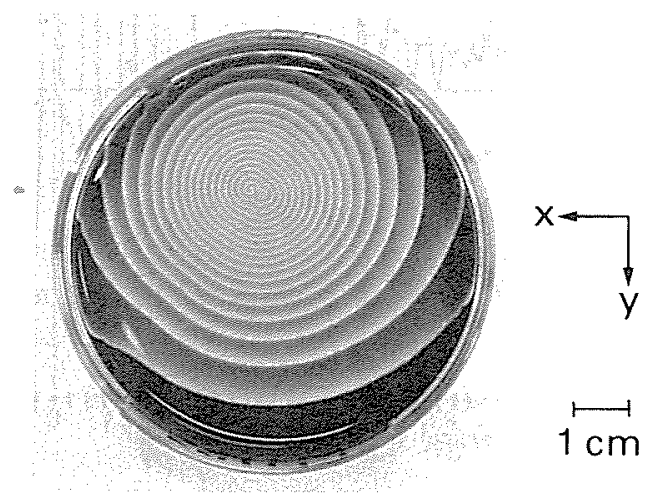

FIG. 1. Pair of spiral waves in a thin BZ reaction layer (depth $0.85 \mathrm{~mm}$ ). Transmitted light is measured in a $(3 \times 3)$. $\mathrm{mm}^{2}$ section at the dish center. 


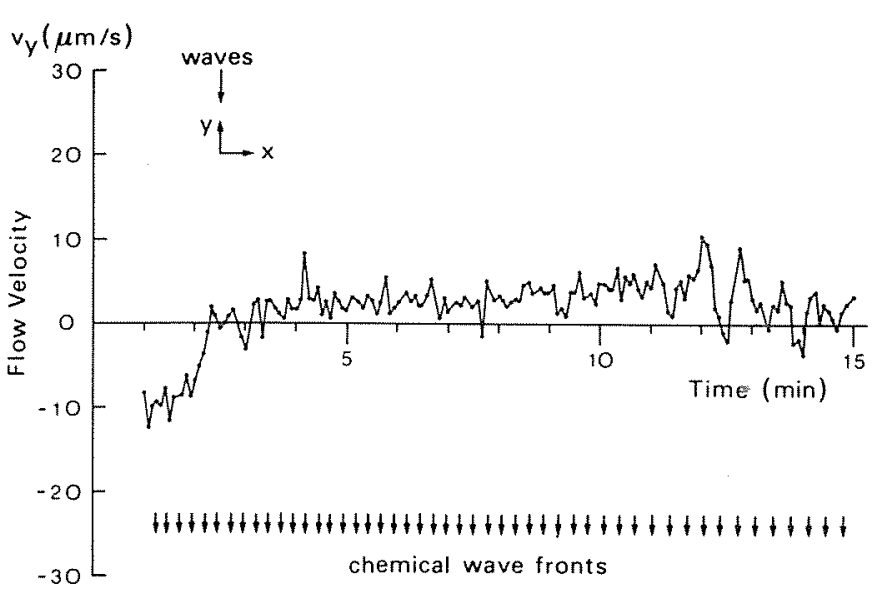

FIG. 2. Temporal trace of flow velocity in the $y$ direction at the center of a covered layer (depth $0.85 \mathrm{~mm}$ ). Arrows are the passage times of wave fronts.

Figure 2 shows the surface flow velocity at the center of a covered dish with a pattern as in Fig. 1. The data parallel to wave propagation indicate that after $3 \mathrm{~min}^{12}$ a small mean flow $\left(v_{y} \approx 4 \mu \mathrm{m} / \mathrm{s}\right)$ is established and after $12 \mathrm{~min}$ an enhanced fluctuation occurs [maximum $\left.v_{y} \approx 10 \mu \mathrm{m} / \mathrm{s}\right]{ }^{12}$ In the perpendicular component $\left(v_{x}\right)$, we do not find any significant flow velocity. Thus, the mean flow has an anisotropic nature: it tends to be directed antiparallel to wave propagation in this geometrical spiral configuration. ${ }^{9}$ The corresponding temporal development of wave profiles, observed in a $(3 \times 3)-\mathrm{mm}^{2}$ outer section of the spirals (center of Fig. 1) shows a remarkable change, just when the enhanced fluctuations in flow velocity occur. An elongated, faint "patchlike" line

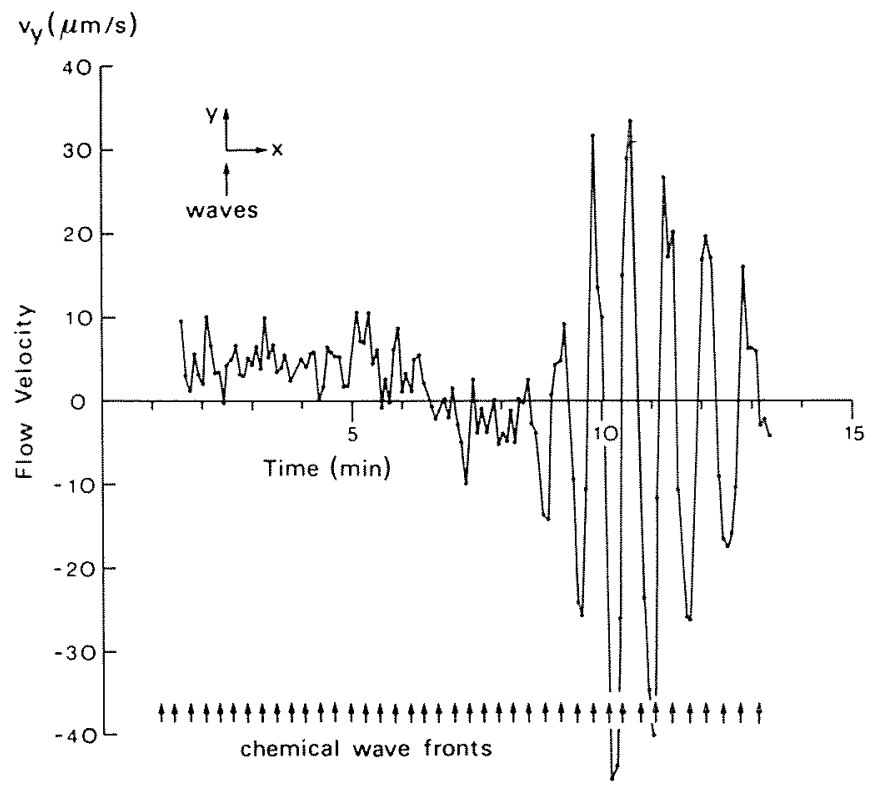

FIG. 3. Temporal trace of flow velocity in the $y$ direction at the center of an uncovered layer (depth $0.85 \mathrm{~mm}$ ). Cover removed at $t=2$ min. Arrows as in Fig. 2 . appears on top of the continuously broadening wave crests and two cycles of periodic weak deformation of the wave profiles are recognized (compare Fig. 4 below).

If the same experiment is performed without a glass cover, an oscillatory hydrodynamic flow is excited spontaneously in the $y$ direction after 9 min (Fig. 3). Its maximum velocity $(\approx 40 \mu \mathrm{m} / \mathrm{s})$ is comparable to the propagation speed of the waves $(\approx 50 \mu \mathrm{m} / \mathrm{s}),{ }^{13}$ and its period $(\approx 40 \mathrm{~s})$ is about twice as large as that of the passage of chemical-wave trains through the detection area. The amplitude of $v_{x}$ is about 3 times smaller. As detected by varying the focal plane of the microscope, the flow amplitudes decrease rapidly with increasing distance from the layer surface.

The temporal development of the waves in the center of the uncovered dish demonstrates (Fig. 4): (i) the wave geometry remains rather stable until $t=10 \mathrm{~m}$ [Fig. 4(a)]; (ii) at $t \approx 11$ min patchlike bright edges appear on the wave crests [open arrows, Fig. 4(b)]; and (iii) periodic deformations from sharp [Fig. 4(f)] to flat [Fig. 4(h)] and back to sharp wave profiles [Fig. 4(i)] follow. These are accompanied by weak geometric distortions with a period close to that of the oscillatory flow ( $\approx 40$ s). In Fig. 4 no scattering particles illuminated by laser light were used, but fortunately we detected a

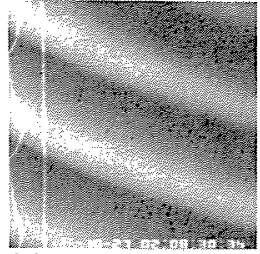

(a) $t=8: 30$

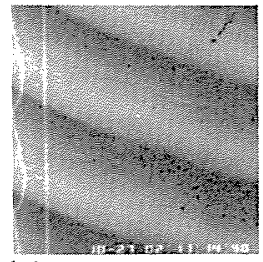

(d) $t=11: 14$

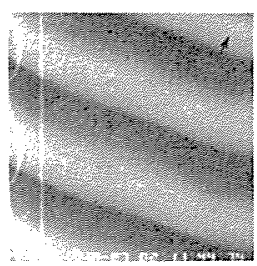

(g) $t=11: 44$

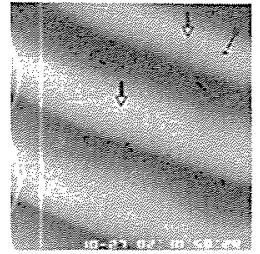

(b) $t=10: 50$

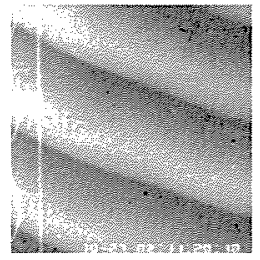

(e) $t=11: 20$

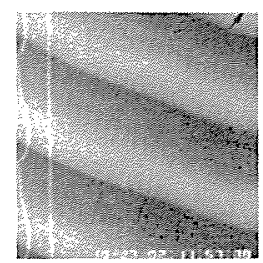

(h) $t=11: 57$

$\longmapsto \mathrm{mm}$

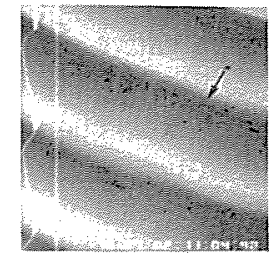

(c) $t=11: 04$

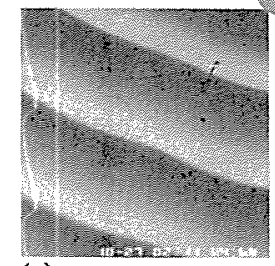

(f) $t=11: 34$

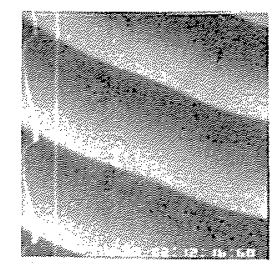

(i) $\mathrm{t}=12: 16$
FIG. 4. Image sequence of the temporal development of waves at $490 \mathrm{~nm}$ under the uncovered condition (without illuminated polystyrene particles). Intensity profiles along the vertical straight line are displayed at the left margins. 
dust particle (size, $50 \mathrm{~mm}$ ) on the layer surface that served as a flow indicator. Black (full) arrows in Fig. 4(b) $-4(\mathrm{i})$ indicate the periodically changing direction of its motion. This corroborates the existence of flow oscillations near the surface and the strong correlation between these oscillations and periodic wave deformations. The thin patchlike edges [Fig. 4(b)] appear to be an early indication for the onset of these flow oscillations. ${ }^{14}$

The wave deformations were further analyzed by monitoring local light intensities in a small area (diameter $<20 \mu \mathrm{m}$ ) with a photodiode in front of the television screen. The time course of such a signal, when observed in a covered dish [Fig. 5(a)], consists of sustained oscillations of transmitted light that are caused by the passage of a periodic chemical-wave train triggered by spiral rotation. The lower envelope increases gradually ndicating a slow increase of ferritin concentration. The amplitude decreases slightly until $t=10 \mathrm{~min}$. At $t \approx 11$ min a small anomaly occurs, which coincides with the time stage of the enhanced fluctuation in flow velocity (Fig. 2). The corresponding power spectrum [Fig. 5(b)] taken during the second half of the measuring time ( 400 to $800 \mathrm{~s}$ ) has its main peak at $f_{0} \approx 0.06 \mathrm{~Hz}$, corresponding to the fundamental frequency of wave passage, while higher harmonics such as $2 f_{0}$ and $3 f_{0}$ are a result of the nonsinusoidal shape of the wave profiles.

In spiral waves under open-air conditions a slow modulation (period about $250 \mathrm{~s}$ ) of the amplitude of the
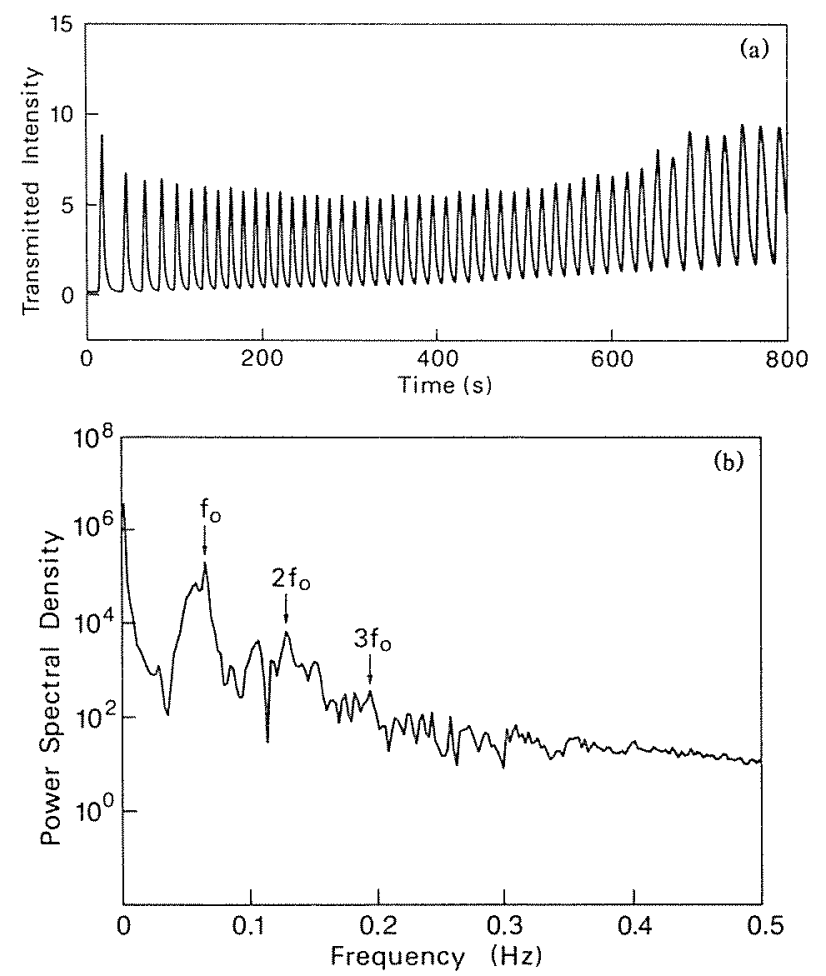

FIG. 5. (a) Time dependence of local transmitted light intensity and (b) its power spectrum in spiral waves under the covered condition (depth $0.94 \mathrm{~mm}$ ). local oscillations in transmittance is recognized [Fig. 6(a)]. Further experiments indicate that, if the depth of the solution layer is increased, the modulation period decreases. Most interestingly, at $t \approx 9 \mathrm{~min}$, the amplitude starts to change periodically with a period twice that of the fundamental frequency $f_{0}$. In the power spectrum [Fig. 6(b)], based again on the second half of the measurements of Fig. 6(a), the period-doubling component $f_{1}\left(=f_{0} / 2\right)$ clearly emerges.

In summary, our investigations reveal periodically changing deformations of chemical-wave profiles in a BZ solution layer, typically 9-12 min after spiral wave initiation. Only two or three periods of weak deformation occur in a covered dish, but pronounced and prolonged periodic deformations are observed in an uncovered dish. They are strongly correlated with hydrodynamic motion close to the liquid/gas interface of the layer. In fact, in an uncovered experiment we detect after 9 min the spontaneous onset of oscillatory hydrodynamic flow which takes place mainly close to the layer surface. The experiments suggest a period-doubling entrainment of flow oscillations by periodic passage of chemical-wave trains.

Recently, we obtained direct evidence that under covered conditions the propagation of singular wave fronts is accompanied by hydrodynamic flow which alternates its direction suddenly when the wave passes through a given point in space. ${ }^{15}$ This supports the idea that chemical waves themselves are able to induce a hy-
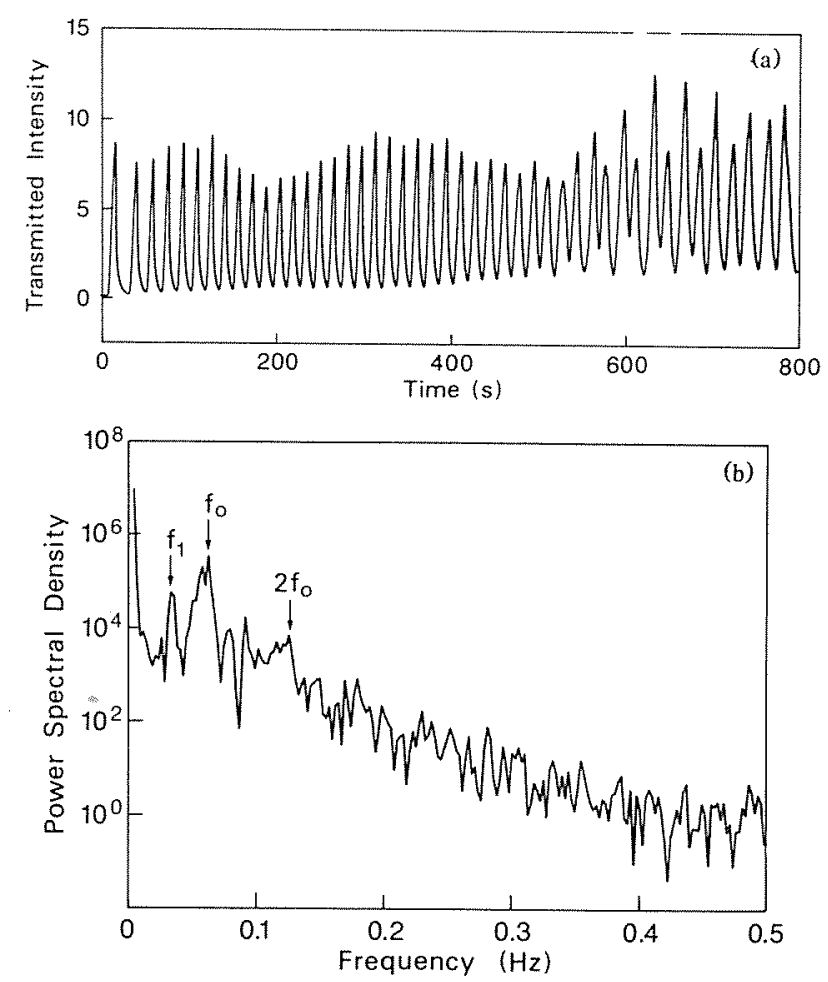

FIG. 6. (a) Time dependence of local transmitted light intensity and (b) its power spectrum in spiral waves with an open liquid/air interface (depth $0.94 \mathrm{~mm}$ ). 
drodynamic instability and wave trains are likely to generate a mean flow (Fig. 2). There are indications that they are also an important source of flow oscillations (together with wave deformations) observed even in a covered dish, and further experimental verification is under way. Consequently, for flow oscillations in an open dish, bulk convection caused by evaporative cooling may well come into play, but does not constitute the main reason. Convection, as well as the exothermicity of the chemical reaction and inhomogeneities in chemical composition have to then be taken into account.

On the other hand, the experiments show that the dynamic wave deformations are a consequence of the oscillatory surface flow. The deformations have an effect on the velocity of wave propagation because they result in variations of curvature of wave fronts ${ }^{16}$ which also modify the flow velocity. ${ }^{15}$ This provides a possible mechanism on how chemically induced flow velocity couples back to the geometric properties of chemical waves. Similar arguments may contribute to an explanation of order-disorder transitions in chemical patterns, when the deformations become strong enough to disrupt the wave fronts. ${ }^{3,10,17}$ A quantitative analysis of such turbulent structures, including the possibility of deterministic chaos, is in progress.

We thank Th. Plesser for his helpful discussions and U. Heidecke, K. Dreher, E. Schlüter, G. Shulte, and B. Plettenberg for their technical assistance. This work was supported by the Stiftung Volkswagenwerk, Hannover.

\footnotetext{
(a) Permanent address: Department of Electrical Engineering, Yamaguchi University, Ube, 755 Japan.
}

${ }^{1}$ Oscillations and Traveling Waves in Chemical Systems, edited by R. J. Field and M. Burger (Wiley, New York, 1985); C. Vidal and P. Hanusse, Int. Rev. Phys. Chem. 5, 1 (1986).

${ }^{2}$ A. M. Zhabotinskii and A. N. Zaikin, J. Theor. Biol. 40, 45 (1973); M. Orban, J. Am. Chem. Soc. 102, 4311 (1980); K. Showalter, J. Chem. Phys. 73, 3735 (1980).

${ }^{3}$ K. I. Agladze, V. I. Krinski, and A. M. Petsov, Nature (London) 308, 834 (1984).

${ }^{4}$ P. M. Wood and J. Ross, J. Chem. Phys. 82, 1924 (1985).

${ }^{5}$ S. C. Müller, Th. Plesser, and B. Hess, Ber. Bunsenges. Phys. Chem. 89, 654 (1985).

${ }^{6}$ C. Normand, Y. Pomeau, and M. G. Velarde, Rev. Mod. Phys. 49, 581 (1977).

${ }^{7} \mathrm{H}$. Linde, in Convective Transport and Instability Phenomena, edited by J. Zierep and H. Oertel, Jr. (Karlsruhe, Braun, 1982), p. 265.

${ }^{8} \mathrm{~S}$. Chandrasekhar, Hydrodynamic and Hydromagnet Stability (Clarendon, Oxford, 1961).

${ }^{9}$ H. Miike, S. C. Müller, and B. Hess, Chem. Phys. Lett. 144, 515 (1988).

${ }^{10}$ S. C. Müller, Th. Plesser, and B. Hess, Naturwissenschaften 73, 165 (1986).

${ }^{1}$ S. C. Müller, Th. Plesser, and B. Hess, Physica (Amsterdam) 24D, 71,87 (1987).

${ }^{12}$ While the sequence of events is reproducible, starting times and durations depend sensitively on the experimental conditions (e.g., ambient temperature, sample preparation).

${ }^{13}$ Under the current experimental conditions the propagation speed $v_{p}$ of waves decreases with time $t$ ( $\mathrm{min}$ ) according to $v_{p}=-1.6 t+72 \mathrm{~mm} / \mathrm{s}$ ( $4 \mathrm{~min}<t<16 \mathrm{~min}$; compare with Ref. $11)$.

${ }^{14}$ It was already suggested in Ref. 11 that the origin of such patches may be attributed to the hydrodynamic flow.

${ }^{15}$ H. Miike, S. C. Müller, and B. Hess, to be published.

${ }^{16}$ Y. Kuramoto, Prog. Theor. Phys. 63, 1885 (1980); J. P. Keener and J. J. Tyson, Physica (Amsterdam) 21D, 307 (1986).

${ }^{17}$ M. Markus, S. C. Müller, Th. Plesser, and B. Hess, Bi Cybernet. 57, 187 (1987). 